\title{
Transitioning From Resistance Devices To Photonic Devices For Temperature Measurements
}

\author{
Zeeshan Ahmed, Gregory Strouse \\ Thermodynamic Metrology, Physical Measurement Laboratory \\ National Institute of Standards and Technology \\ Maryland, United States of America \\ \{zeeshan.ahmed, gregory.strouse\}@nist.gov
}

\begin{abstract}
For the past century, industrial temperature measurements have relied on resistance measurement of a thin metal wire or filament whose resistance varies with temperature. Though resistance thermometers can routinely measure industrial temperatures with uncertainties of $10 \mathrm{mK}$, they are sensitive to mechanical shock which causes the sensor resistance to drift over time requiring frequent off-line, expensive, and time consuming calibrations. These fundamental limitations of resistance thermometry have produced considerable interest in developing photonic temperature sensors to leverage advances in frequency metrology and to achieve greater mechanical and environmental stability. We are developing a suite of photonic devices that leverage advances in microwave and $\mathrm{C}$-band light sources to fabricate cost-effective photonic temperature sensors. Our preliminary results indicate that using photonic devices such as the ring resonator we can measure short term temperature fluctuations of $80 \mu \mathrm{K}$ at room temperature. Photonic sensor technology provides a low cost, lightweight, portable and electromagnetic interference (EMI) resistant solution which can be deployed in a wide variety of settings ranging from controlled laboratory conditions, a noisy factory floor, advanced manufacturing, to the variable environment of a residential setting.
\end{abstract}

Keywords - photonics; thermometry; fiber Bragg grating; ring resonator; sapphire whispering gallery mode resonator

\section{INTRODUCTION}

Temperature is second most measured quantity in the world, second only to time. Temperature measurements play a central role in every aspect of modern life ranging from process control in advance manufacturing to environmental control and monitoring in buildings and automobiles. $[1,2]$ For the last 100 years, temperature measurements have relied on resistance measurement of a metallic wire whose resistance varies with temperature.[3, 4] Industrial resistance thermometers can measure temperatures with uncertainties of $10 \mathrm{mK}$ meeting the metrology needs of an overwhelming majority of the market.[5] Humidity and residual impurities can hasten oxidation at higher temperatures, resulting in hysteresis.[4, 6] Furthermore, mechanical shock and thermal stress can strain the sensing element, causing the sensor resistance to drift over time. Consequently, resistance thermometers require expensive, time consuming calibrations on a periodic basis which significantly increases their ownership costs.[3] These fundamental limitations of resistance thermometry have produced considerable interest in the development of photonic temperature sensors as an alternative to resistance thermometers[7-9] as they have the potential to leverage recent advances in frequency metrology to provide greater temperature sensitivity while being robust against drift caused by environmental shock.

Photonic temperature sensors exploit temperature dependent changes in a material's properties. Change in material properties directly impacts resonant frequency of light-matter interactions, e.g. absorption .[10-12] Photosensitive dyes such as the green florescent protein (GFP) have been utilized to generate thermal maps. Single cell thermal mapping with GFP proteins is being developed as an assay for evaluating the efficacy of photo-thermal cancer therapies. Similarly, changes in ro-vibrational levels of gaseous molecules have been probed using spectroscopic techniques including absorption spectroscopy[13], Raman scattering[14], and Brillouin scattering[15]. Absorption spectroscopy approaches have focused on the development of fast scanning laser based system to measure temperature dependent changes in band frequency and band width[13]. The direct absorption approach has been successfully used to probe temperature and pressure changes in gas flows under extreme conditions such as a shock tube.[16, 17] Several national laboratories around the world are utilizing absorption spectroscopy (Doppler induced bandwidth broadening) to measure the Boltzmann constant. $[13,18,19]$

Absorption spectroscopy approaches, under development, are well-suited to laboratory use in illuminating the role of temperature gradient in chemical processes however, at the present cost, complexity, and lack of portability of such techniques limit deployment opportunities in industrial settings. To meet the temperature sensing needs of the industrial sector, researchers have focused on the development of fiber-optic and chip based photonic sensors[11, 20, 21]. These devices typically utilize interferometry techniques to detect temperature induced changes in substrate's (silica or silicon) refractive index and physical dimensions. Temperature sensitivities of up to $720 \mathrm{pm} / \mathrm{K}$ have been reported for selectively coated photonic crystal fibers[22]. The small form factor of optical devices e.g. an optical fiber is conducive to the deployment of sensor in a wide variety of 
environments. Fiber Bragg gratings (FBG), commercially available from several vendors, e.g. have found uses in down bore applications.

Photonic Temperature Sensor Research: Recognizing the disruptive potential of photonic temperature sensor technology, we have launched research program aimed at developing novel photonic temperature sensors that provide sufficient accuracy to satisfy the metrology needs of the present and emerging markets. We are focused on leveraging the low cost of light sources used in telecommunications industry (microwave and infrared) to develop affordable, readily deployable, sensors fabricated using commercially relevant technologies. In this note, the discussion is limited to three of our on-going thermometry projects: the whispering gallery mode resonator (WGMR), fiber Bragg gratings (FBG) and the ring resonator (RR).

Whispering Gallery Mode Resonator: For the last 40 years, WGMRs have been utilized as ultra-stable resonators (fractional frequencies $<10^{-12}$ ).[23] Although WGMRs have been fabricated with a wide variety of materials including fused quartz and glass spheres, mono-crystalline synthetic sapphire has emerged as a material of choice as it boasts modes with quality-factors of a million.[23, 24] Considerable effort has been expended in developing an athermal WGMR for the express purpose of providing a stable frequency source.[25, 26] The temperature dependence of WGMR derives from the thermo-optic effect, the intrinsic temperature dependence of the refractive index (or permittivity) of the sapphire crystal can be expressed as:

$\frac{1}{f \mathrm{o}}\left(\frac{\partial f \mathrm{o}}{\partial \mathrm{T}}\right)=\frac{1}{f \mathrm{o}}\left\{\partial \frac{f \mathrm{o}}{\varepsilon \perp} \frac{\partial \varepsilon \perp}{\partial \mathrm{T}}+\frac{\partial f \mathrm{o}}{\partial \varepsilon \|} \frac{\partial \varepsilon \|}{\partial \mathrm{T}}+\frac{\partial f \mathrm{o}}{\partial \mathrm{L}} \frac{\partial L}{\partial \mathrm{T}}+\left(\frac{\partial f \mathrm{o}}{\partial \mathrm{a}}\left(\frac{\partial \mathrm{a}}{\partial \mathrm{T}}\right)\right)\right\}(1)$

Where, $f_{o}$ is the center resonance frequency, $\varepsilon_{\perp}$ is the permittivity in the radial direction, $\varepsilon_{\|}$is the permittivity in the axial direction, $L$ is the axial length of the sapphire disk, and $a$ is the sapphire disk diameter with the assumption that the relative magnetic permeability is exactly one. The whispering gallery resonances occur in the sapphire disk when standing waves are excited along the circumference of the disk with minimal reflection losses. Reflection, absorption, and scattering losses are the dominant energy dissipation pathways. For the quasi-TM modes of the WGMR, Eq. 1 may be reduced to estimate the frequency-temperature dependence relationship:

$$
1 / f o\left(\frac{\left(\frac{\partial f o}{\partial \mathrm{T}}\right)\left(-\frac{1}{2 \varepsilon \|}\right)}{\frac{\delta \varepsilon\|\|}{\delta \mathrm{T}}}-\alpha \perp\right.
$$

Where $\alpha_{\perp}$ is the thermal expansion in the radial direction. The thermo-optic effect in sapphire is $\approx 100$ times larger than its thermal expansion coefficient, hence only the thermo-optic effect needs to be considered to describe the temperature dependent response of sapphire WGMR thermometers. The large temperature dependence of sapphire WGM's frequency and the ease of measuring peak frequency of high $-\mathrm{Q}$ modes has enabled us to develop a highly sensitive and accurate temperature sensor based on a disk of monocrystalline

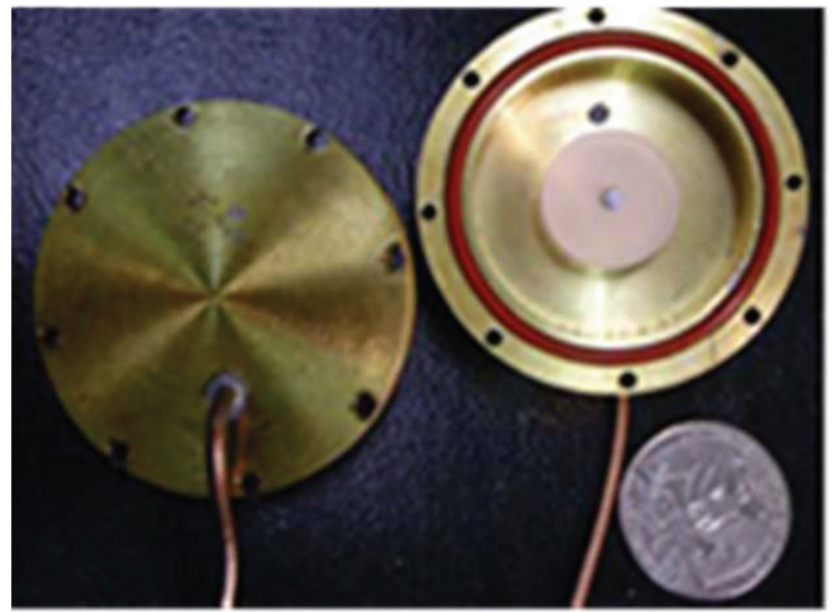

Figure 1: Sapphire disk resonator based WGMR have been demonstrated to measure temperature with uncertainties of $10 \mathrm{mK}$ or better over the temperature range of 273 to $373 \mathrm{~K}$

sapphire to demonstrate temperature measurement uncertainty $(k=2)$ of $10 \mathrm{mK}$ between $273.15 \mathrm{~K}$ and 373 K.[24] Sapphire WGMR thermometers with spherical and cylindrical geometries have also been demonstrated. We are currently, developing a WGMR thermometer based on a sapphire tube resonator where the sapphire resonator is mechanically stabilized with metallic collar (Fig 2). Compact, cylindrical device geometry, similar to modern Platinum resistance thermometers is necessary to minimize end-user disruption.

Fiber Bragg gratings: FBGs are narrow band filters commonly used in the telecommunications industry for routing information. FBG are fabricated using photo-sensitive optical fibers (e.g $\mathrm{H}_{2}$ loaded $\mathrm{Ge}$ doped fibers) that are exposed to spatially varying UV light. The spatially varying UV light field is created by either using a deep UV laser in a Michelson interferometer or passing a broadband UV source through a hard mask. The periodically varying light field modifies the local structure of silica, creating a periodic variation in the local refractive index that acts like a Bragg grating.[7, 10, 11] Wavelength of light resonant with the Bragg period is reflected back, while non-resonant wavelengths pass through the grating. The grating equation is given by:

$$
\lambda=2 * n e * \Lambda \quad(3)
$$

Change in surrounding temperature impacts the effective grating period due to linear thermal expansion of the optical fiber and its refractive index due to temperature (thermo-optic effect).

$\Delta \lambda=2 *\left\{\left(\frac{\partial n e}{\partial l}\right)+\left(n e\left(\frac{\partial \Lambda}{\partial}\right)\right\}+2 *\left\{\Lambda\left(\frac{\partial n e}{\partial T}\right)+n e\left(\frac{a}{\partial T}\right)\right\}\right.$

In fused silica, the thermo-optic effect is a factor of ten larger than thermal expansion, hence the contribution of thermal expansion in the Bragg wavelength shift can be ignored. Existing literature indicates FBG show a temperature dependent shift of $\approx 10 \mathrm{pm} / \mathrm{K}$ over the temperature range of $243 \mathrm{~K}$ to $393 \mathrm{~K},[7,8,10,11]$ although there is disagreement on whether the FBG temperature response is linear or quadratic. We have embarked on a research program that 


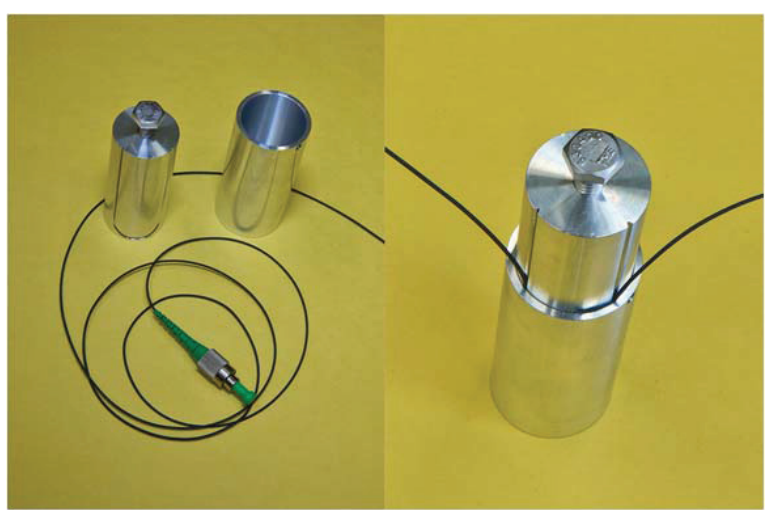

Figure 2: Aluminum block consisting of a cylinder and holster captures a FBG between the two metal surfaces ensuring uniform sensor heating.

seeks to carefully characterize the temperature response of a strain-free FBG, identify and quantify the impact of possible confounding variables such as humidity and strain, and present an uncertainty budget along with recommended calibration procedure that would enable accurate temperature measurement. For our preliminary examinations we fabricated a specially designed aluminum comparison block (Fig 2) to ensure uniform heating of the sensor area. Our preliminary results (Fig 3), using commercially available FBGs indicate that over the temperature range of $243 \mathrm{~K}$ to $283 \mathrm{~K}$, the FBG response $(9-10 \mathrm{pm} / \mathrm{K})$, when modeled as a linear function yields a quadratic residual similar to that observed by Flockhart et. al.[27] However, we note that these preliminary measurements lack sufficient frequency accuracy to definitely determine if the residual is indicative of higher order structure. We are currently building a dedicated laser based FBG interrogation setup to investigate the temperature and pressure dependent response of FBG sensors. This system integrates a high speed wavemeter to allow accurate determination of wavelength, reducing our wavelength measurement uncertainty by up to a factor of 50 .

Ring Resonators: As an alternative to macroscopic FBG sensors, we are developing ring resonators as on-chip, portable temperature sensors. In recent years, ring resonator[28, 29] based devices have been exploited for bio-chemical sensing applications.[21, 30] In these sensors, temperature induced shifts in resonance frequency have been a complicating feature that adversely impacts sensor sensitivity and specificity. Indeed, development of an athermal ring resonator is an active area of research.[31, 32] The temperature dependence of the ring resonator arises from temperature-induced changes in refractive index (n) and in the physical dimensions of the ring. A qualitative analysis of a ring resonator yields a resonance wavelength for a single ring resonator of:

$$
\lambda=[\lambda * n e * L] / m
$$

Where, $\lambda$ is the vacuum wavelength, $\mathrm{n}_{\mathrm{e}}$ is the effective refractive index, $\mathrm{m}$ is the mode number, $\mathrm{L}$ is the ring perimeter, and $\mathrm{T}$ is the temperature. Thus, the temperatureinduced shift in wavelength is given by:

$$
\lambda=\left\{\left[\left(\frac{\partial n}{\partial T}\right)+n\left(\frac{\partial L}{\partial T}\right)\left(\frac{1}{L}\right)\right]\left(\frac{1}{n g}\right)\right\} *\left(\Delta \mathrm{T} * \lambda_{\mathrm{m}}\right)
$$

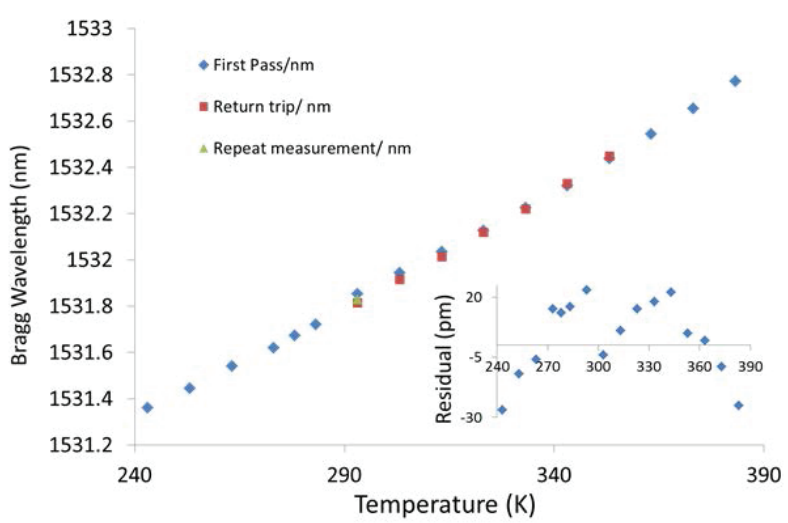

Figure 3: NIST preliminary results from an investigation of the temperature dependent response of FBG appears to show a linear dependence. The insert shows the residual between linear fit and experimental data.

Where the group index is $n_{g}=\left[\mathrm{n}-\lambda_{\mathrm{m}}\left(\partial \mathrm{n} / \partial \lambda_{\mathrm{m}}\right)\right]$ The variation in the refractive index due to the thermal expansion coefficient for silicon $\left(\sim 1 \times 10^{-6} / \mathrm{K}\right)$ is a factor of 100 smaller than the silicon thermo-optic effect $\left(1 \times 10^{-4} / \mathrm{K}\right)$. Thus, the silicon ring resonator's thermal response dominates its strain response. We recently demonstrated (Fig 4) that silicon-based optical ring resonator device with a ring diameter of $11 \mu \mathrm{m}$ and gap (between waveguide and ring) of $130 \mathrm{~nm}$ can resolve temperature differences of $1 \mathrm{mK}$ using the traditional wavelength scanning methodology.[1] An even lower noise floor of $80 \mu \mathrm{K}$ for measuring temperature difference is achieved in the side-of-fringe, constant power mode measurement. In this measurement scheme which we refer to as the "side of fringe, constant power mode", the laser power is maintained at a constant power level and its frequency centered on the side of resonance at the point of steepest descent (point of half-contrast). A small, temperaturedependent shift in resonance frequency results in a large change in transmitted laser intensity. Since the resonance lineshape is known, intensity fluctuations can be converted to an estimate of center frequency change without measuring the peak shape. Our recent results indicate that at room temperature the "side of fringe constant power mode" can resolve temperature difference of $80 \mu \mathrm{K}$ at a temporal resolution of one second. Furthermore, the passivating $\mathrm{SiO}_{2}$

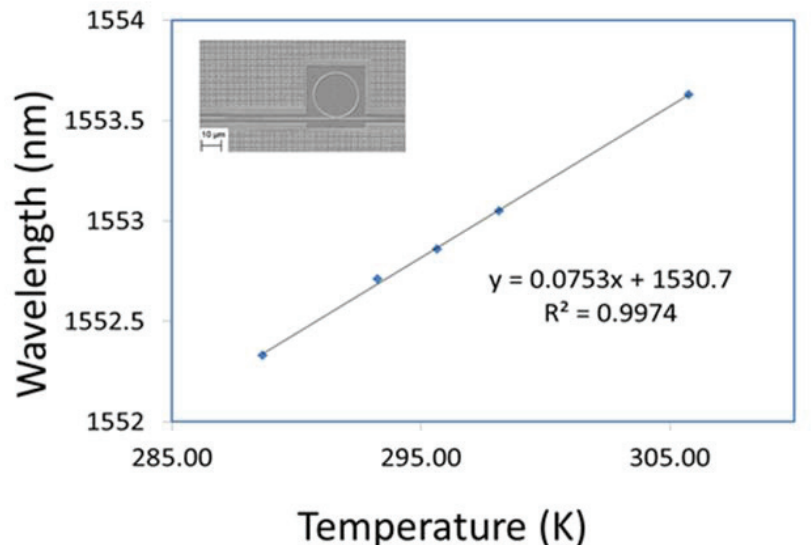

Figure 4: A ring resonator device with an 11 micrometer radius shows a temperature dependent shift in resonance of $77 \mathrm{pm} / \mathrm{K}$ 
layer on the photonic chip makes the sensor virtually impervious to humidity changes, removing a common confounding variable that inhibits resistance thermometry.[1] Incorporating the use of frequency stabilized lasers can further lower the noise floor. We are currently working on optimizing the ring resonator thermometer to improve its temperature sensitivity over the temperature range of $200 \mathrm{~K}$ to $550 \mathrm{~K}$.

Summary: The fundamental limitations of resistance temperature device namely their sensitivity to mechanical shock, humidity and oxidation have fostered significant interest in the development of photonic temperature sensors as an alternative. FBG have already found a niche in down-bore applications with researchers looking to expand the market to medical devices. In recent years we have demonstrated that telecom industry light sources in the microwave and infrared can be harnessed to develop cost-effective photonic temperature measurement solutions such as the sapphire WGMR and the on-chip ring resonator. These devices have been demonstrated to provide temperature measurement capabilities that are on par, if not better than traditional resistance measurement devices.

\section{ACKNOWLEDGMENT}

We would like to thank Jay Hendricks and Sunil Mittal for helpful discussions

\section{REFERENCES}

[1] H. Xu, M. Hafezi, J. Fan, J. Taylor, G. F. Strouse, and Z. Ahmed, "Ultra-Sensitive Chip-Based Photonic Temperature Sensor Using Ring Resonator Structures," Optics Express, vol. 22, pp. 30983104, 2014.

[2] F. A. Jolesz, "MRI-Guided Focused Ultrasound Surgery," Annual Review of Medicine, vol. 60, p. 417, 2009.

[3] G. F. Strouse, "Standard Platinum Resistance Thermometer Calibrations fromthe Ar TP to the Ag FP," NIST Special Publication 250-250-81, 2008.

[4] R. Price, "The Platinum resistance Thermometer," Platinum Metals Rev., vol. 3, pp. 78-87, 1959

[5] A. I. Pokhodun, "Current status and prospects for development of thermometry," AIP Conf. Proc., vol. 89, p. 1552, 2013.

[6] B. W. Mangum, "Stability of Small Industrial Platinum Resistnace Thermometers," Journal of Research of the NAtional Bureau of Standards, vol. 89, pp. 305-316, 1984.

[7] S. J. Mihailov, "Fiber Bragg Grating Sensors for Harsh Environments," Sensors, vol. 12, pp. 1898-1918, 2012.

[8] A. D. Kersey and T. A. Berkoff, "Fiber-optic Bragg-grating differential-temperature sensor," Photonics Technology Letters, IEEE, vol. 4, pp. 1183-1185, 1992.

[9] R. Yun-Jiang, D. J. Webb, D. A. Jackson, Z. Lin, and I. Bennion, "In-fiber Bragg-grating temperature sensor system for medical applications," Lightwave Technology, Journal of, vol. 15, pp. 779$785,1997$.

[10] J. Hecht, Understanding Fiber Optics 4th ed.: Prentice Hall, 2002.

[11] D. A. Krohn, Fiber Optic Sensors: Fundamentals and Applications 3ed.: ISA, 2000.

[12] F. L. Walls and D. W. Allan, "Measurements of Frequency Stability," Proceeding of the IEEE, vol. 74, pp. 162-168, 1986.

[13] C. Daussay, M. Guinet, A. Amy-Klein, and KHermier, "Direct Determination of the Boltzmann Constant by an Optical Method," Phy. rev. Lett, vol. 98, pp. 250801-250801-4, 2007.
[14] Z. Xu, D. Liu, H. Liu, Q. Sun, Z. Sun, X. Zhang, et al., "Design of distributed Raman temperature sensing system based on singlemode optical fiber," Front. Optoelectron. China, vol. 2, pp. 215218,2009 .

[15] L. Zou, X. Bao, and L. Chen, "Simultaneous distributed Brillouin strain and temperature sensor with photonic crystal fiber," Proceedings of SPIE vol. 5384, pp. 13-17, 2004.

[16] A. Farooq, J. B. Jeffries, and R. K. Hanson, "CO2 concentration and temperature sensor for combustion gases using diode-laser absorption near 2.7 mm," Applied Physics B vol. 90, pp. 619-628, 20008.

[17] A. Farooq, J. B. Jeffries, and R. K. Hanson, "Sensitive detection of temperature behind reflected shock waves using wavelength modulation spectroscopy of $\mathrm{CO} 2$ near $2.7 \mathrm{~mm}$," Applied Physics B, vol. 96, pp. 161-173, 2009.

[18] K. Djerroud, C. Lemarchand, A. Gauguet, C. Daussay, S. Briaudeau, B. Darquie, et al., "Measurement of the Boltzmann constant by the Doppler broadening technique at a $3.8 \times 10-5$ accuracy level," Comptes Rendus Physique, 2009.

[19] L. Moretti, A. Castrillo, E. Fasci, M. D. D. Vizia, G. Casa, G. Galzerano, et al., "Determination of the Boltzmann Constant by MEans of Precision Measurements of $\mathrm{H}_{2}{ }^{18} \mathrm{O}$ Line Shapes at 1.39 mm," Physical Reveiw Letters, vol. 111, pp. 060803-060803-5, 2013.

[20] G.-D. Kim, H.-S. Lee, C.-H. Park, S.-S. Lee, B. T. Lim, H. K. Bae, et al., "Silicon photonic temperature sensor employing a ring resonator manufactured using a standard CMOS process," Opt. Express, vol. 18, pp. 22215-22221, 2010.

[21] M.-S. Kwon and W. H. Steier, "Microring-resonator-based sensor measuring both the concentration and temperature of a solution," Opt. Express, vol. 16, pp. 9372-9377, 2008.

[22] Y. Peng, J. Hou, Z. Huang, and Q. Lu, "Temperature sensor based on surface plasmon resonance within selectively coated photonic crystal fiber," Applied Optics, vol. 51, pp. 6361-6367, 2012.

[23] S. A. Savchenkov, A. B. Matsko, V. S. Ilchenko, N. Yu, and L. Maleki, "Whispering-gallery-mode resonators as frequency references. II Stabilization," J. Opt. Soc. Am. B, vol. 24, pp. 29882997, 2007.

[24] G. F. Strouse, "Sapphire Whispering Gallery Thermometer," International Journal of Thermophysics, vol. 28, pp. 1812-1821, 2007.

[25] D. L. Creedon, M. E. Tobar, and J.-M. Le Floch, "Single-crystal sapphire resonator at millikelvin temperatures: Observation of thermal bistability in high-Q factor whispering gallery modes," Phys. Rev. B, vol. 82, pp. 104305-104305-5, 2010.

[26] R. Boudot, C. Rocher, N. Bazin, S. Galliou, and V. Giordano, "High-precision temperature stabilization for sapphire resonators in microwave oscillators," Reveiw of Scientific Instruments, vol. 76, pp. 095110-095110-6, 2005.

[27] G. M. H. Flockhart, R. R. J. Maier, J. S. Barton, W. N. MacPherson, J. D. C. Jones, K. E. Chisholm, et al., "Quadratic Behavior of Fiber Bragg Grating Temperature Coefficients," Applied Optics, vol. 43, pp. 2744-2751, 2004.

[28] W. W. Rigrod, "The Optical Ring Resonator," The Bell System Technical Journal, pp. 907-916, 1965.

[29] L. Stern, I. Goykhman, B. Desiatov, and U. Levy, "Frequency locked micro disk resonator for real time and precise monitoring of refractive index," Opt. Lett., vol. 37, pp. 1313-1315, 2012.

[30] X. Tu, J. Song, T.-Y. Liow, M. K. Park, J. Q. Yiying, J. S. Kee, et al., "Thermal independent Silicon-Nitride slot waveguide biosensor with high sensitivity," Opt. Express, vol. 20, pp. 2640$2648,2012$.

[31] B. Guha, B. B. C. Kyotoku, and M. Lipson, "CMOS-compatible athermal silicon microring resonators," Opt. Express, vol. 18, pp. 3487-3493, 2010 .

[32] B. Guha, K. Preston, and M. Lipson, "Athermal silicon microring electro-optic modulator," Opt. Lett., vol. 37, pp. 2253-2255, 2012. 